\title{
NeUROPSICOLOGÍA ORGANIZACIONAL: NUEVA PERSPECTIVA EN LA GESTIÓN DEL TALENTO HUMANO
}

\section{Sinuhé Carlos Guardado López ${ }^{a}$}

Recibido: 12 de julio, 2019 - Aprobado: 9 de septiembre, 2019

Cómo citar: Guardado López, G. L. (2019). Neuropsicología organizacional: nueva perspectiva en la gestión del talento humano. Ciencia, Economía y Negocios, 3(1), 49-57. Doi: https://doi.org/10.22206/ceyn.2019.v3i1.pp49-57

\section{Resumen}

En la actualidad, los avances científicos y la influencia teórica como práctica de las neurociencias para las ciencias del comportamiento son indiscutibles, por tanto es importante redimensionar al ser humano y al trabajador en las organizaciones. Por décadas, en la literatura de la ciencia de la administración, especificamente en el área de recursos humanos, ha prevalecido el paradigma ambientalista con respecto al comportamiento del trabajador. Sin embargo, como fue señalado, las neurociencias y el avance de otras ciencias como la neuropsicología, exigen al administrador y a las personas que gestionan trabajadores en las organizaciones —incluso desde el proceso de selección - replantearse los paradigmas que se han conformado en torno a la productividad, la motivación, la toma de decisiones y el comportamiento de los empleados. El presente artículo exhibe sólidos sustentos cientificos que permitirán refutar la visión ambientalista del trabajador que ha prevalecido en el área de recursos humanos. Además, hará posible entender a la persona $y$ al trabajador como un ser en el que confluyen tres esferas: la biológica, la psicológica y la cultural. Lo antes planteado es de utilidad para los gerentes, pues les ayuda a entender y comprender los cimientos de las llamadas competencias laborales.

Palabras clave: recursos humanos; comportamiento organizacional; comportamiento humano; neuropsicología.

Códigos JEL: M12, M14, M54.

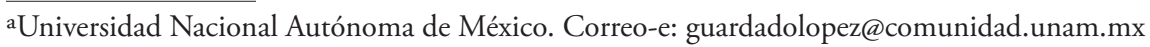

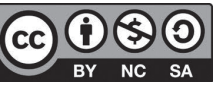




\title{
Organizational NeUROPSYCHOLOGY: A NEW PERSPECTIVE IN THE MANAGEMENT OF HUMAN TALENT
}

\author{
Sinuhé Carlos Guardado López
}

Received: July 12, 2019, 2019 • Approved: September 9, 2019

\begin{abstract}
Scientific advances and the theoretical as a practical influence of neurosciences for Behavioral Sciences are indisputable in recent times; for this reason, it is important to resize the human being and the worker in organizations. For decades, in the literature of the Science of Administration, specifically in the area of Human Resources, the environmental paradigm has prevailed over the behavior of the worker. However, as noted, the neurosciences and the advance of other sciences, such as Neuropsychology, require the administrator and the people who manage workers in organizations, even from the selection process, rethink the paradigms that have been formed around productivity, motivation, decision making and employee behavior. This article shows solid scientific support that will refute the environmentalist vision of the worker, which has prevailed in the area of Human Resources, and will allow us to understand the person and the worker as a being in which three spheres converge: the biological one, psychological and cultural. This is useful for managers to understand the foundations of the so-called labor competencies.
\end{abstract}

Key words: Human resources; organizational behavior; human behavior; Neuropsychology.

JEL Codes: M12, M14, M54. 


\section{Introducción}

Por lo general, en las universidades — sobre todo en las carreras universitarias relativas a las ciencias administrativas - y en los diferentes centros de trabajo se sobreentiende que el comportamiento de la persona se manifiesta únicamente por factores que lo motivan o afectan y que tienen un origen exógeno al trabajador.

En el presente artículo se pretende dejar de manifiesto lo señalado, indicando que la forma de gestionar al personal o al área de Recursos Humanos se ha modificado, teniendo en la actualidad un enfoque de competencias, las cuales se sustentan en los diversos circuitos o las distintas funciones y subfunciones neuropsicológicas que poseen todas las personas y, consecuentemente, los trabajadores de toda organización.

Si el trabajador no tiene bien desarrollados los circuitos o sus respectivas funciones y subfunciones neuropsicológicas, es imposible que cuente con las competencias señaladas en una descripción de puestos, ya que las funciones neuropsicológicas conforman los cimientos de tales competencias laborales.

\section{La ciencia de la administración y los recursos humanos}

La evolución que han experimentado las organizaciones durante el siglo $\mathrm{xx}$ y en las dos primeras décadas del siglo XXI, aunado a diversas variables tecnológicas, económicas y políticas, ha modificado la forma de gestionar las organizaciones.

Lo anterior ha influido en la filosofía institucional de las organizaciones $y$, consecuentemente, en la forma en que se deben cumplimentar los objetivos en el dinámico y competitivo ambiente en el que estas se desenvuelven. Por lo tanto, una de las tareas que más ocupan a la ciencia de la Administración y a los gerentes es la forma en que las organizaciones captan sus recursos y cómo los gestionan.

Desde hace tiempo, en la ciencia de la Administración —en Latinoamérica-, particularmente en el área de Recursos Humanos, ha prevalecido una perspectiva ambientalista en torno a la productividad y al comportamiento del trabajador; es decir, se considera que prácticamente 
todo lo positivo y lo negativo que influye en él y su rendimiento laboral proviene de factores exógenos al individuo, ya sea familia, clima laboral, relaciones interpersonales dentro y fuera del centro de trabajo, motivaciones extrínsecas, problemas "personales" que se generan fuera del alcance del trabajador y que lo aquejan, etc.

No obstante, contadas veces en las organizaciones -incluso desde la educación técnica, o bien, universitaria- se considera que los problemas de comportamiento y de productividad del trabajador o del técnico y/o profesionista en preparación, según sea el caso, provienen de aspectos biológicos, singularmente, neuropsicológicos.

Por lo general, se otorga una sobrevaloración a los factores externos a la persona o al trabajador; se afirma casi siempre que la merma en su rendimiento proviene de circunstancias que lo rodean, reflejándose las consecuencias de su ambiente en su comportamiento.

\section{La gestión del talento humano}

El recurso más importante que tienen las organizaciones, pero a la vez el más complejo de estudiar, comprender y explicar, es el denominado talento humano, entendido como la aptitud o capacidad que tiene una persona para el desempeño de algo (Real Academia Española, 2019).

Tomando como base el concepto de talento humano es ineludible definir uno de los términos que ha cobrado importancia recientemente: la gestión del talento humano, comprendida como el proceso de trabajo que requiere de la coordinación de esfuerzos y del talento de las personas que integran una organización. Estos elementos serán encaminados eficazmente hacia un objetivo determinado.

La gestión del talento humano es una tarea compleja, aunque generalmente poco apreciada, pues de forma banal en diversas organizaciones se considera una labor que cualquier licenciado en Administración, Psicología, Relaciones Industriales, Contaduría o cualquier carrera afín a las ciencias administrativas. No obstante, es una ardua encomienda que precisa de un personal altamente calificado y con conocimientos científicos sobre el comportamiento humano. 
En ocasiones, resulta extraño y hasta anecdótico para los jóvenes que se incorporan al mercado laboral el poco esmero que diversas organizaciones imprimen a sus procesos de selección de personal, caracterizándose por la premura y la somera forma con que se lleva a cabo tal proceso, aunado a la poca experiencia de las personas que ejercen tal labor, al ser muy jóvenes o con poca calificación, teniendo únicamente educación universitaria; pocos de ellos tienen estudios de posgrado o son coordinados por personas con un mayor nivel educativo.

No es de extrañar que algunos de los nuevos colaboradores en las organizaciones ni siquiera tengan un contrato firmado, no hayan entregado completamente la documentación requerida, o bien, no concluyeran en forma correcta un proceso de selección, que suele ser somero; aun así, ya se encuentran laborando en la organización.

Conforme a lo referido, se puede afirmar que una de las tendencias que se ha presentado en las últimas dos décadas es la gestión del talento humano por competencias o gestión de Recursos Humanos por competencias. Empero, para poder dimensionar a la neuropsicología organizacional y contextualizar su impacto es obligatorio responder qué son las competencias, qué son las competencias laborales, de qué forma las competencias se relacionan con la neuropsicología y qué es la neuropsicología organizacional.

Como respuesta a la primera pregunta, se puede señalar que las competencias, de forma general, son las características de personalidad que devienen en comportamientos y que influyen en el desempeño exitoso de una persona en su puesto de trabajo (Alles, 2015).

De manera específica, las competencias laborales son un elenco de competencias (generales) de las que dispone un empleado para desarrollar de forma adecuada sus funciones en el empleo. Las competencias laborales comprenden conocimientos generales y específicos, habilidades técnicas, calificaciones tradicionales. Esto brindará al empleado la capacidad para enfrentar y resolver con éxito situaciones inciertas, nuevas e irregulares en la vida laboral. Por lo tanto, la competencia laboral no es solo el desempeño productivo por medio de la instrucción, también es aprendizaje significativo y aprendizaje por experiencia en situaciones concretas de trabajo (Ducci, 1997). 
La relación entre la neuropsicología y las competencias probablemente no es fácil de identificar a primera vista para los profesionales de la Administración y la Psicología organizacional; pero, la experiencia de algunos especialistas en el área neuropsicológica y de la administración, precisamente en recursos humanos, permite evidenciar tal relación, ya que las diversas funciones neuropsicológicas que las personas desarrollan a través del tiempo son los cimientos de las competencias laborales y académicas.

En el entorno organizacional es evidente la importancia de gestionar el talento humano, subrayando que un sistema de gestión es "(...) un conjunto de políticas, conceptos y prácticas coherentes entre sí, con el propósito de alcanzar objetivos organizacionales de la empresa de manera eficiente y eficaz" (Chiavenato \& Villamizar, 2009, p. 1). Mientras que el concepto de talento resulta en patrones de pensamiento recurrentemente consistentes o comportamiento (Lewis \& Heckman, 2006).

Lo enunciado permite plantear una gestión estratégica del talento humano. Este proceso comprende el conjunto de cesiones y acciones directivas enfocadas hacia la gestión de personas que trabajan en una organización, quienes poseen múltiples conocimientos que no pueden tratarse de forma separada de la dimensión social del trabajador; su correcta aplicación derivará en un mejoramiento continuo durante la planeación, la implementación y el control de las estrategias organizacionales (Cuesta, 2010).

\section{Neuropsicología organizacional y gestión del talento humano}

Conforme a lo expuesto, se puede responder a la pregunta ¿qué es la neuropsicología organizacional? Afirmando que es la disciplina que mediante los conocimientos e instrumentos científicos de diversas ciencias base - como la neuropsicología, la psicología organizacional y la administración - coadyuva a la óptima gestión estratégica del talento humano por medio de la comprensión e integración de los conocimientos, las experiencias, las actitudes, las aptitudes y los comportamientos del personal, los cuales se traducen en las competencias personales y laborales de los trabajadores.

La neuropsicología organizacional es un paso necesario en la comprensión y el entendimiento del comportamiento y desempeño del 
talento humano; considerar de forma aislada el componente biológico de la persona sería una falacia y una visión miope ante el recurso más valioso, pero a la vez más complejo que posee la organización, pues incluye actitudes, aptitudes, conocimientos y experiencias que derivan en múltiples comportamientos en un puesto de trabajo.

Se puede sustentar la importancia de una correcta toma de decisiones por parte de un supervisor, o bien, por parte de un directivo; no obstante, en ocasiones, difícilmente se sabe y se comprende lo que está detrás del iceberg de comportamientos que posee un trabajador y que se reflejan en determinada decisión y en su desempeño como tal; es decir, ¿qué motiva a un trabajador, supervisor o directivo a tomar determinado curso de acción?, ¿existe alguna influencia biológica o neuropsicológica al respecto?

Lo planteado puede sustentarse científicamente mediante las funciones ejecutivas, las cuales comprenden los procesos implementados para planear y organizar la conducta, la inhibición de conductas inapropiadas para la realización de una tarea y el mantenimiento de un pensamiento flexible durante la resolución de problemas. De igual forma, se integra el comportamiento propositivo, el cual se constituye por las conductas que se encaminan a cumplir un objetivo determinado y nos ayuda a resolver ciertas situaciones en concreto (Guardado, 2018; Ostrosky et al., 2003; Matute, Roselli, Ardila, y Ostrosky, 2014; Portellano, 2005).

Adicionalmente, se puede referir el planteamiento que desde hace más de dos décadas hizo Goleman (1998), quien acotaba que numerosos directivos se quejaban de la ausencia de ciertas competencias (devenidas en comportamientos) en sus trabajadores; por ejemplo, la falta de aptitudes sociales de los trabajadores, por lo que también buscaban en un trabajador capacidad de escuchar y comunicarse verbalmente, adaptabilidad y creatividad, autocontrol, confianza y motivación, eficacia grupal e interpersonal, cooperación, capacidad de trabajar en equipo y habilidad de negociación, participación activa en la organización y liderazgo.

Por lo señalado, en el vertiginoso mundo de las organizaciones las bases neuropsicológicas son relevantes debido a que las exigencias organizacionales y las demandas laborales actuales requieren el establecimiento de metas a corto plazo, que deberán alcanzarse de forma adecuada, rápida y con la menor inversión de tiempo y recursos. 


\section{Conclusión}

El conocimiento y entendimiento del personal en un proceso de gestión estratégica del talento humano permitiría mediante las bases neuropsicológicas, incluso a los llamados gerentes de línea — quienes siempre han tenido de forma implícita la gestión del personal- (Dessler \& Varela, 2017) colocar a la persona indicada en el puesto correcto, apuntalando la selección científica del personal; además, se mejoraría el desempeño laboral de cada uno, se desarrollarían con un sustento neurocientífico las capacidades y las habilidades de cada individuo, se fomentaría y mantendría un buen estado anímico en el área u organización y se protegería la salud y la condición física de los trabajadores.

Si una organización desea ser competitiva es importante saber leer y anticipar las señales que emiten el mercado y el entorno organizacional. Uno de los fundamentos que les permitirá a las organizaciones hacer frente a la dinámica del entorno es el reto de la mejora constante de las competencias de sus trabajadores en todos los niveles.

Lo antes expuesto exige una labor constante con soporte neuropsicológico para actualizar los conocimientos, optimizar las habilidades y experiencia, mejorar las actitudes y los comportamientos; esto en conjunto con una dirección estratégica eficaz y una adecuada filosofía institucional que permitirá la consecución de los objetivos organizacionales a largo plazo (Oltra et al., 2005).

Finalmente, por lo expuesto en este artículo es imperioso e innegable que los administradores, expresamente los encargados del área de Recursos Humanos y los integrantes de la alta Dirección, den los pasos necesarios hacia un nuevo entendimiento comportamental del talento humano con el que cuentan en la organización.

Los avances de las neurociencias son indiscutibles, por lo que es evidente la necesidad de superar la prevaleciente idea de los factores exógenos como los únicos influyentes en el comportamiento de los trabajadores, idea que ha prevalecido por décadas en la literatura de la ciencia de la administración, que se enfoca en la influencia ambiental sobre el individuo, sin aceptar y entender de forma adecuada las bases neuropsicológicas del trabajador. 


\section{Referencias}

Alles, M. (2015) Selección por competencias. México, D.F.: Granica.

Chiavenato, I. \& Villamizar, G. (2009). Gestión del talento humano. Recuperado de www.academia.edu/download/34860731/LECTURA_CLASE1_Gestion_del_Talento_Humano.doc

Cuesta. A. (2010) La gestión del talento humano y del conocimiento. Bogotá: Ediciones ECOE.

Dessler, G. \& Varela, R. (2017). Administración de recursos humanos: un enfoque latinoamericano. ( $6^{\mathrm{a}}$ ed.) México, D.F.: Pearson.

Ducci, M. A. (1997). El enfoque de competencia laboral en la perspectiva internacional. Formación basada en competencia laboral: situación actual y perspectivas, 15-26.

Ducci, M. A. (1998). La Inteligencia Emocional en la Empresa. Barcelona, España: Kairós.

Guardado, S. C. (2018). Neuropsicología: su importancia en el comportamiento del trabajador. Iberoamerican Business Journal. 1(2), 49-59. doi: http://dx.doi.org/10.22451/5817.ibj2018.vol1.2.11010

Lewis, R. E. \& Heckman, R. J. (2006). Talent management: A critical review. Human resource management review, 16(2), 139-154.

Matute, E., Roselli, M., Ardila, A. \& Ostrosky, F. (2014) ENI - 2 Evaluación Neuropsicológica Infantil Manual de Aplicación. México, D.F.: Manual Moderno.

Oltra, V., Curós, M., Díaz, C., Rodríguez, J., Teba, R. \& Tejero, J. (2005). Desarrollo del factor humano. Barcelona, España: Editorial UOC.

Ostrosky-Solís, F., Gómez, E., Matute, Rosselli, M., Ardila, A. \& Pineda, D. (2003) Neuropsi Atención y Memoria 6 a 85 años. México, D.F.: American Book Store.

Portellano, J. (2005). Introducción a la Neuropsicología. México, D.F.: Mc Graw Hill.

Real Academia Española. (2019). Talento. Recuperado de https://dle.rae. es/?id=YzSnsTL

Santos, A. C. (2010). Gestión de talento humano y del conocimiento. Bogotá, Colombia: Ecoe Ediciones. 